\title{
Dark energy and extending the geodesic equations of motion: its construction and experimental constraints
}

\author{
Achilles D. Speliotopoulos
}

Received: 21 April 2009 / Accepted: 27 December 2009 / Published online: 23 January 2010

(C) The Author(s) 2010. This article is published with open access at Springerlink.com

\begin{abstract}
With the discovery of Dark Energy, $\Lambda_{\mathrm{DE}}$, there is now a universal length scale, $\ell_{\mathrm{DE}}=c /\left(\Lambda_{\mathrm{DE}} G\right)^{1 / 2}$, associated with the universe that allows for an extension of the geodesic equations of motion. In this paper, we will study a specific class of such extensions, and show that contrary to expectations, they are not automatically ruled out by either theoretical considerations or experimental constraints. In particular, we show that while these extensions affect the motion of massive particles, the motion of massless particles are not changed; such phenomena as gravitational lensing remain unchanged. We also show that these extensions do not violate the equivalence principal, and that because $\ell_{\mathrm{DE}}=14010_{820}^{800} \mathrm{Mpc}$, a specific choice of this extension can be made so that effects of this extension are not be measurable either from terrestrial experiments, or through observations of the motion of solar system bodies. A lower bound for the only parameter used in this extension is set.
\end{abstract}

Keywords Dark energy · Geodesic equations of motion

\section{Introduction}

The recent discovery of Dark Energy (see [1,2] and references therein) has broadened our knowledge of the universe, and has demonstrated once again that it can hold surprises for us. This discovery has, most assuredly, also brought into sharp relief the degree of our understanding of the universe. In this paper, we will study one specific

\footnotetext{
A. D. Speliotopoulos $(\varangle)$

Department of Physics, University of California, Berkley, CA 94720, USA

e-mail: ads@berkeley.edu
}

A. D. Speliotopoulos

Department of Mathematics, Golden Gate University, San Francisco, CA 94105, USA 
implication of this discovery: with the discovery of Dark Energy, $\Lambda_{\mathrm{DE}}$, there is now a universal length scale, $\ell_{\mathrm{DE}}=c /\left(\Lambda_{\mathrm{DE}} G\right)^{1 / 2}, 1,2$ associated with the universe that allows for extensions of the geodesic equations of motion (GEOM). We find that contrary to expectations, these extensions are not automatically ruled out by theoretical considerations, nor are they ruled out by experimental constraints either through terrestrial experiments or through solar system tests of general relativity. Indeed, we show in this paper that one specific extension of the GEOM is a viable alternative to the GEOM, and we obtain a lower bound for the only free parameter used in its construction, a power-law exponent, $\alpha_{\Lambda}$.

There are good theoretical and physical reasons for studying the range of extensions of the geodesic equations of motion that are allowed. Arguments for the use of the geodesic equations of motion to describe the motion of massive test particles in curved spacetime are based on various statements of the equivalence principle, and the principle of general covariance (see chapter 4 of [5]), along with arguments in favor of simplicity and aesthetics. Importantly, these arguments are made in addition to those made in favor of Einstein's field equation. Namely, these is no unique way of deriving the geodesic equations of motion from the field equations. Indeed, in 1938 Einstein, Infield and Hoffman attempted to show that as a consequence of the field equations, massive test particles will travel along geodesics in the spacetime [6]. These attempts have continued to the present day $[7,8]$.

Extensions and modifications of the GEOM have been made before, of course. On the level of Newtonian dynamics, Modified Newtonian Dynamics (MOND) [9-11] has been proposed as an alternate explanation of the galactic rotation curves. On the relativistic level, there has been recent efforts [12] to develop a general framework to study modifications to the GEOM in the weak field, linearized gravity limits. The major impetus for this work has been to describe a series of dynamical anomalies- the Pioneer anomaly (see [13] and [14]), the flyby anomaly [15], and the lengthening of the Astronomical Unit [16]— that have been observed at the Solar system scale.

The focus of this paper is to establish the underlying theoretical framework that can be used to describe structures and dynamics at the galactic length scale and above. In a future paper (A. D. Speliotopoulos, in preparation) this framework will be applied to an analysis of the galactic rotation curves, and the impact that this extension has on phenomena at cosmological length scales will be studied. As such, we focus here on the Dark Energy energy length scale, and on how the existence of this scale allows for extensions of the GEOM. Indeed, we find that with this length scale, $\ell_{\mathrm{DE}}$, extensions of the GEOM are not difficult to construct. The quotient $c^{2} R / \Lambda_{\mathrm{DE}} G$ is dimensionless, and functionals of this quotient can easily be used to extend the GEOM. What is more relevant is whether or not the resultant equations of motion will be a physically viable alternative to the GEOM. As such we will be guided in our extension of the GEOM by the four conditions listed below. They are deliberately chosen to be conservative in scope, and thus stringent in their application. Somewhat surprisingly, we will show that there is at least one extension of the GEOM that satisfies all four.

\footnotetext{
1 Our $\ell_{\mathrm{DE}}$ differs from the length scale, $r_{\Lambda}$, defined in [3] by a factor of $\sqrt{3}$.

2 It is also possible to construct the length scale $\left(\hbar c / \Lambda_{\mathrm{DE}}\right)^{1 / 4} \approx 85 \mu \mathrm{m}$. Experiments have shown that this scale does not affect the Newtonian potential [4].
} 
First, we require that the extension preserve the equivalence principle, which is one of the underlying principles on which general relativity is founded. In the following sections of the paper, we will explicitly see that this preservation is assured by the fact that $\ell_{\mathrm{DE}}$ is the same for all test particles. This universal nature of $\ell_{\mathrm{DE}}$ is crucial. While other length scales - say, the proton mass - could be used for the extension, the resultant equations of motion would depend on this mass. They could not then be applied to the motion of protons without explicitly violating the uniqueness of free fall condition.

Second, we require that the extension not change the equations of motion for massless test particles; such particles must still follow the GEOM. All astronomical observations - of which gravitational lensing is playing an increasingly important role — are based on the motion of photons of various wavelengths. Modifications to the equations of motion for photons will require a reinterpretation of these observations, a daunting step not to be taken without good reason. We will show that by considering a class of extensions that is based on conformally scaling the rest mass of the test particle, we arrive at extended GEOMs that, on the one hand, will not change the motion of massless test particles, but will, on the other, change the motion of massive ones. Being conformal, the motion of photons will not be affected by this class of extensions, and they will still travel along null geodesics; phenomena such as gravitational lensing will remain unchanged. While in form this class of extensions resembles a scalar field theory that is non-minimally and nonlinearly coupled to the scalar curvature, $R$, such theories are constructed at the quantum level. Our extension of the GEOM is done at the classical, $\hbar=0$, level, with the scale of the coupling set by $\ell_{\mathrm{DE}}$.

The third condition involves the attempts [6-8] at proving that the GEOM are the unique consequence of the Einstein field equations (see also p. 72 of [5]). These proofs would seem to rule out any physically relevant extension of the GEOM, and by necessity, our extension of the GEOM cannot be precluded by such proofs. That the extension is possible is because these proofs focus on the motion of test particles in regions where the Einstein tensor, $G_{\mu \nu}$, vanishes. We will see that in these regions the extended GEOM reduce to the GEOM, and thus do not violate these proofs. Indeed, we will explicitly construct the energy-momentum tensor for an inviscid fluid of massive particles propagating under the extended GEOM.

The fourth condition is the most stringent of the four. With the exception of the as-yet unexplained anomalies described above, we require that the extension of the GEOM not produce effects that are measurable either in terrestrial experiments, or through the motion of bodies in the solar system that have traditionally been used to test general relativity. While stringent, we will nevertheless show explicitly that a choice of extension can be made which satisfies it. Physically, this choice is possible because at $\left(7.21_{-0.84}^{+0.83}\right) 10^{-30} \mathrm{~g} / \mathrm{cm}^{3}[17], \Lambda_{\mathrm{DE}}$ is orders of magnitude smaller than the density of matter, $\rho_{\text {limit }}$, either currently achievable in terrestrial experiments (where densities exceed $10^{-18} \mathrm{~g} / \mathrm{cm}^{3}$ ), or present in the solar system (where the density of matter in Mercury's orbit exceeds $10^{-23} \mathrm{~g} / \mathrm{cm}^{3}$ ). Correspondingly, at $14010_{820}^{800} \mathrm{Mpc}$ $\ell_{\mathrm{DE}}$ is more than three times larger than the observed size of the universe, and is orders of magnitude larger than the solar system. Nevertheless, we find that even though the disparity between the magnitude of $\Lambda_{\mathrm{DE}}$ and $\rho_{\text {limit }}$-or, equivalently, between $\ell_{\mathrm{DE}}$ and the size of the solar system-is large, a nonlinear function of $c^{2} R / \Lambda_{\mathrm{DE}} G$ is needed 
in constructing the extension for its effects not to have already been seen in terrestrial experiments. The simplest of these extensions has only one free parameter, $\alpha_{\Lambda}$, a power-law exponent that determines the behavior of the function at densities both much larger than $\Lambda_{\mathrm{DE}}$, and much smaller than it. Lower bounds for $\alpha_{\Lambda}$ are set by requiring that the extension does not produce observable effects in current terrestrial experiments.

While it may be possible to apply the analysis in this paper to the explanation of Solar system anomalies such as the Pioneer anomaly, the focus of this research is on phenomenon at the galactic scale or longer. It is for this reason that we require our extension to be constrained only by experiments and observations that are currently well-understood, and for which the underlying physics is well-known. We leave to future work the question of whether or not our analysis can be applied to explaining the Pioneer and other Solar-system-scale anomalies.

\section{Extending the Geodesic Lagrangian}

We begin our extension of the GEOM with Einstein's field equation in the presence of a cosmological constant

$$
R_{\mu \nu}-\frac{1}{2} g_{\mu \nu} R+\frac{\Lambda_{\mathrm{DE}} G}{c^{2}} g_{\mu \nu}=-\frac{8 \pi G}{c^{4}} T_{\mu \nu}
$$

where $T_{\mu \nu}$ is the energy-momentum tensor for matter, $R_{\mu \nu}$ is the Ricci tensor, Greek indices run from 0 to 3 , and the signature of $g_{\mu \nu}$ is $(1,-1,-1,-1)$. While there is currently no consensus as to the nature of Dark Energy (proposals have been made that identify it with the cosmological constant $\Lambda_{\mathrm{DE}}$ [3], with quintessence [18-20], or even as a consequence of loop quantum cosmology [21]), modifications to Einstein's equations to include the cosmological constant are well known and are minimal. We will thus identify Dark Energy with the cosmological constant in this paper, and require only that $\Lambda_{\mathrm{DE}}$ changes so slowly that it can be considered a constant in our analysis.

Requiring that Eq. (1) still holds under the extension of the GEOM is a choice, one which, we will see below, is the simplest. Although it may seem surprising that we can still make this choice even though we will be changing the GEOM, extensions of the GEOM need not change the relation between $R_{\mu \nu}$ and $T_{\mu \nu}$ given in Eq. (1). They can rather change the precise form that $T_{\mu \nu}$ takes for matter. To see this, consider the following.

The total action, $S$, for a system consisting of gravity, radiation, and matter can be written as a sum of three parts: $S=S_{\text {grav }}+S_{\text {radiation }}+S_{\text {matter }}$. Here, $S_{\text {grav }}$ is the action for gravity, $S_{\text {radiation }}$ is the action for radiation, and $S_{\text {matter }}$ is the action for matter. We will show below that the class of extended GEOM we consider here will not change the equations of motion for radiation so that $S_{\text {radiation }}$ will not be changed. The extension will certainly change $S_{\text {matter }}$, however, and in the next section we will explicitly construct the energy-momentum tensor for an inviscid fluid whose constituents follow an extended GEOM. For the present argument, we only need to note that whatever the form taken for $S_{\text {matter }}$, we are still free to choose $S_{\text {grav }}$ to be the Hilbert action; Eq. (1) then follows after taking the functional derivative of $S$ with respect to the metric. 
This ability to change $S_{\text {matter }}$ while leaving $S_{\text {grav }}$, and thus Eq. (1), unchanged was explicitly exploited in the construction of minimally coupled scalar fields, $\phi_{R}$. There, the mass term of the scalar field, $m^{2} \phi_{R}^{2}$, is replaced by $R m^{2} \phi_{R}^{2}$ in the action for matter, and yet $S_{\text {grav }}$ is still taken to be the Hilbert action (see Sect. II.D). Einstein's field equations, Eq. (1), still hold; the only change is the form that $T_{\mu \nu}$ takes.

Both the geodesic Lagrangian

$$
\mathcal{L}_{0} \equiv m c\left(g_{\mu \nu} \frac{d x^{\mu}}{d t} \frac{d x^{\nu}}{d t}\right)^{1 / 2}
$$

and the GEOM

$$
v^{v} \nabla_{\nu} v^{\mu} \equiv \frac{D v^{\mu}}{\partial t}=0
$$

(where $v^{\mu}=\dot{x}^{\mu}$ is the four-velocity of the test particle), have natural geometric meaning. The first is a proper time interval, while the second is the equation for parallel transport, which determines the shortest time-like path connecting two points. But, aside from their inherent geometric meaning, there is also a good physical reason to take Eq. (3) as the equations of motion for a test particle. In the absence of Dark Energy, Eq. (3) is the most general form that a second-order evolution equation for a test particle can take which still obeys the equivalence principle.

Any extension of $\mathcal{L}_{0}$ would require a dimensionless, scalar function of some fundamental property of the spacetime folded in with some physical property of the universe. In our homogeneous and isotropic universe, there are few opportunities to do this. A fundamental vector certainly does not exist in the spacetime, and while there is a scalar (the scalar curvature, $R)$ and three tensors $\left(g_{\mu \nu}\right.$, the Riemann tensor, $R_{\mu \nu, \alpha} \beta$, and the Ricci tensor, $\left.R_{\mu \nu}\right), R_{\mu \nu, \alpha}^{\beta}$ has units of inverse length squared. While it is possible to construct a dimensionless scalar $m^{2} G^{2} R / c^{4}$ for the test particle, augmenting $\mathcal{L}_{0}$ using a function of this scalar would introduce additional forces that will depend on the mass of the test particle, and thus violate the uniqueness of free fall principle. It is also possible to construct the scalar $g_{\mu \nu} v^{\mu} v^{\nu} / c^{2}$, but because of the mass-shell condition, $v_{\mu} v^{\mu}=c^{2}$, any such extension of $\mathcal{L}_{0}$ will not change the GEOM. Scalars may also be constructed from $R_{\mu \nu}$ and powers of $R_{\mu \nu, \alpha}{ }^{\beta}$ by contracting them with the appropriate number of $v^{\mu} / c$ 's, but these scalars will once again have dimension of inverse length raised to some power, and, as with the Ricci scalar, once again a rest mass $m$ is needed to construct the dimensionless quantity.

The situation changes dramatically in the presence of Dark Energy. With a universal length scale, $\ell_{\mathrm{DE}}$, it is now possible to construct from the Riemann tensor and its contractions dimensionless scalars of the form,

$$
\frac{c^{2} R}{\Lambda_{\mathrm{DE}} G}, \frac{R_{\mu \nu} v^{\mu} v^{\nu}}{\Lambda_{\mathrm{DE}} G}, \frac{c^{2} v^{\mu} v^{\nu}}{\left(\Lambda_{\mathrm{DE}} G\right)^{2}} R_{\mu \alpha, \beta \gamma} R_{\nu}^{\alpha, \beta \gamma}, \frac{v^{\mu} v^{\nu} v_{\gamma} v_{\delta}}{\left(\Lambda_{\mathrm{DE}} G\right)^{2}} R_{\mu \alpha, \nu \beta} R^{\gamma \alpha, \delta \beta}, \ldots
$$


Although extensions to $\mathcal{L}_{0}$ can be constructed with any of these terms, we look at extensions with the form:

$$
\mathcal{L}_{\mathrm{Ext}} \equiv m c\left[1+\mathfrak{D}\left(c^{2} R / \Lambda_{\mathrm{DE}} G\right)\right]^{\frac{1}{2}}\left(g_{\mu \nu} \frac{d x^{\mu}}{d t} \frac{d x^{\nu}}{d t}\right)^{\frac{1}{2}} \equiv \mathfrak{R}\left[c^{2} R / \Lambda_{\mathrm{DE}} G\right] \mathcal{L}_{0},
$$

with the implicit condition that $v^{2}=c^{2}$ for massive test particles. We make this choice for the following reasons.

First, having $\Re$ be a function only of $c^{2} R / \Lambda_{\mathrm{DE}} G$ is the simplest extension that can be chosen; other choices will induce velocity-dependent effects in the extended GEOM. Second, we will find below that any extension of $\mathcal{L}_{0}$ of the form Eq. (5) will not change the equations of motion for massless test particles; they can still be reduced to the GEOM. Extensions of the form Eq. (5) will only affect the motion of massive test particles. Third, we require that the extension of the GEOM not produce effects that should have already been seen in terrestrial experiments; these experiments are done in the nonrelativistic and weak gravity limits. Constraints in the choice of $\mathfrak{R}$ are thus found in these limits, where it is clear from Eq. (4) that the second term reduces to the first, while the other terms are higher order in the curvature. We are thus left with $c^{2} R / \Lambda_{\mathrm{DE}} G$ with which to construct an extension of the GEOM.

As $\ell_{\mathrm{DE}}=14010_{820}^{800} \mathrm{Mpc}$, the question remains whether it is possible to use another, shorter length scale in its place to extended the GEOM; this extension could then be used to describe deviations from geodesic motion on shorter length scales. One such application would be in explaining Solar system scale anomalies such as the Pioneer and flyby anomalies. At this scale, a natural length scale would be $M_{\odot} G / c^{2}=$ $1,480 \mathrm{~m}$, where $M_{\odot}$ is the mass of the Sun, and the resultant extension of the GEOM may be applicable to the description of motion on the Solar system scale (see [12]). Its application to the description of motion at the galactic scale or longer is more problematic, however, and it is precisely on these length scales that we are concerned with here. On the galactic scale, stars can be treated as test particles, and as $M_{\odot} G / c^{2}$ depends explicitly on the mass of the Sun, the use of this length scale in extending the GEOM would mean that the motion of stars in galaxies would depend on the mass of the Sun. This would not be physically reasonable, and would also violate the uniqueness of free fall condition.

If $\mathfrak{D}(x)$ is the constant function, then $\mathcal{L}_{\text {Ext }}$ differs from $\mathcal{L}_{0}$ by an overall constant that can be absorbed through a reparametrization of time. Only non-constant $\mathfrak{D}(x)$ are relevant. It is how fast $\mathfrak{D}(x)$ changes that will determine its effect on the equations of motion, and not its overall scale. Indeed, in extending $\mathcal{L}_{0}$ we have in effect performed a conformal scaling of $\mathcal{L}_{0}$ by replacing the constant rest mass $m$ of the test particle with a curvature-dependent rest mass $m \Re\left[c^{2} R / \Lambda_{\mathrm{DE}} G\right]$. All dynamical effects of this extension can therefore be interpreted as the rest energy gained or lost by the test particle due to the local curvature of the spacetime. The scale of these effects is of the order of $m c^{2} / L$, where $L$ is some relevant length scale of the dynamics. The additional forces from $\mathcal{L}_{\text {Ext }}$ are thus potentially very large. For these effects not to have already been seen, $\mathfrak{D}\left(c^{2} R / \Lambda_{\mathrm{DE}} G\right)$ must change very slowly at current limits to experimental measurements. 
As mentioned above, using Einstein's field equations, Eq. (1), was a choice. In particular, notice that because $\mathcal{L}_{\text {Ext }}$ is the result of conformally scaling the rest mass by $\Re$, we may choose to instead reduce $\mathcal{L}_{\text {Ext }}$ to $\mathcal{L}_{0}$ through the conformal transformation of the metric $\tilde{g}_{\mu \nu}=\mathfrak{R}^{1 / 2} g_{\mu \nu}$. However, doing so will result in a Ricci tensor $\tilde{R}_{\mu \nu}$ that is nonlinearly related to $R_{\mu \nu}$ :

$$
\begin{aligned}
\tilde{R}_{\mu \nu}= & R_{\mu \nu}-\frac{1}{2} \nabla_{\mu} \nabla_{\nu} \log \mathfrak{R}-\frac{1}{4} g_{\mu \nu} \nabla_{\alpha} \nabla^{\alpha} \log \mathfrak{R} \\
& +\frac{1}{8} \nabla_{\mu} \log \mathfrak{R} \nabla_{\nu} \log \mathfrak{R}-\frac{1}{8} g_{\mu \nu} \nabla_{\alpha} \log \mathfrak{R} \nabla^{\alpha} \log \mathfrak{R} .
\end{aligned}
$$

where $\nabla_{\mu}$ is the covariant derivative for $g_{\mu \nu}$. As Eq. (1) holds for $R_{\mu \nu}$, it cannot hold for $\tilde{R}_{\mu \nu}$. It will instead be replaced by a nonlinear relation between $\tilde{R}_{\mu \nu}$ and $T_{\mu \nu}$, resulting in a higher-order theory of gravity. Thus, instead of choosing Eq. (1) to hold for the Ricci tensor and extend the GEOM, we could have chosen a higher order theory of gravity from the start while preserving the GEOM. We would argue, however, that making this second choice would change the foundations of classical general relativity - a much more drastic step — that would also result in a less tractable theory.

\subsection{The extended GEOM for massive test particles}

For massive test particles, the extended GEOM from $\mathcal{L}_{\text {Ext }}$ is

$$
\frac{D^{2} x^{\mu}}{\partial t^{2}}=c^{2}\left(g^{\mu \nu}-\frac{v^{\mu} v^{\nu}}{c^{2}}\right) \nabla_{\nu} \log \Re\left[c^{2} R / \Lambda_{\mathrm{DE}} G\right]
$$

where we have explicitly used $v^{2}=c^{2}$. It has a canonical momentum with a magnitude of

$$
p^{2}=p_{\mu} p^{\mu}=m^{2} c^{2}\left[1+\mathfrak{D}\left(c^{2} R / \Lambda_{\mathrm{DE}} G\right)\right]
$$

and the interpretation of $m \Re\left[c^{2} R / \Lambda_{\mathrm{DE}} G\right]$ as an effective rest mass can be readily seen. What also can be readily seen is that $\mathcal{L}_{\text {EXT }}$ and Eq. (7) have lost the geometrical meaning that $\mathcal{L}_{0}$ and Eq. (3) have. Namely, the worldline of a massive test particle is not that which minimizes the proper time between two points; it is instead one that is either attracted to, or repelled from (depending on the choice of $\mathfrak{R}$ ), regions where the scalar curvature is extremized.

The dynamical implications of the new terms in Eq. (7), along with the conditions under which they are relevant, can most easily be seen by noting that $R=4 \Lambda_{\mathrm{DE}} G / c^{2}+$ $8 \pi T G / c^{4}$, where $T=T_{\mu}^{\mu}$. Then $\mathfrak{R}\left[c^{2} R / \Lambda_{\mathrm{DE}} G\right]=\mathfrak{R}\left[4+8 \pi T / \Lambda_{\mathrm{DE}} c^{2}\right]$, where the '4' comes from the dimensionality of spacetime. Thus, in regions of spacetime where either $T_{\mu \nu}=0$ or when $T$ is a constant, the right hand side of Eq. (7) vanishes, and our extended GEOM reduces back to the GEOM. 


\subsection{Dynamics of massless test particles}

For massless test particles, we note that $\mathcal{L}_{\text {Ext }}$ is related to $\mathcal{L}_{0}$ by a scaling of the rest mass. This scaling can be formally interpreted as a conformal transformation of the metric, $g_{\mu \nu}$. As null geodesics are preserved under conformal transformations up to an affine transformation, the extended GEOM is equivalent to the GEOM, and we still have $v^{\nu} \nabla_{\nu} v^{\mu}=0$.

This result can be shown explicitly in the following analysis. Because massless particles follow null geodesics, for these particles we consider extensions of the form

$$
\mathcal{L}_{\mathrm{Ext}}^{\gamma}=\Re\left[c^{2} R / \Lambda_{\mathrm{DE}} G\right] \mathcal{L}_{0}^{\gamma},
$$

where we have taken

$$
\mathcal{L}_{0}^{\gamma}=\frac{1}{2} g_{\mu \nu} \frac{d x^{\mu}}{d t} \frac{d x^{\nu}}{d t}
$$

As usual, the GEOM comes from $\mathcal{L}_{0}^{\gamma}$, while the equations of motion that come from $\mathcal{L}_{\text {Ext }}^{\gamma}$ are

$$
0=\mathfrak{R}\left(\frac{d}{d t} \frac{\delta \mathcal{L}_{0}^{\gamma}}{\delta \dot{x}^{\mu}}-\frac{\delta \mathcal{L}_{0}^{\gamma}}{\delta x^{\mu}}\right)+\frac{\delta \mathcal{L}_{0}^{\gamma}}{\delta \dot{x}^{\mu}} \frac{d \Re}{d t}-\mathcal{L}_{0}^{\gamma} \nabla_{\mu} \Re .
$$

Taking now the mass-shell condition, $\mathcal{L}_{0}^{\gamma}=0$, Eq. (11) reduces to simply

$$
0=\frac{D}{\partial t}\left(\Re \frac{d x^{\mu}}{d t}\right) \text {. }
$$

After the reparametrizing $d t \rightarrow \Re d t$ [5], we arrive at the expected result, $v^{\nu} \nabla_{\nu} v^{\mu}=0$. Importantly, this result means that the usual general relativistic effects associated with photons - the gravitational redshift and the deflection of light—are not effected by our extension of the GEOM.

\subsection{Impact on the equivalence principles}

The statements [22] of the equivalence principal we are concerned with here are the following:

Uniqueness of free fall It is clear from Eq. (7) that the worldline of a freely falling test particle under the extended GEOM does not depend on its composition or structure.

The weak equivalence principle Our extension also satisfies the weak equivalence principle to the same level of approximation as the GEOM. The weak equivalence principle is based on the ability to choose a frame in a neighborhood of the worldline of the test particle where $\Gamma_{\alpha \beta}^{\mu} \approx 0$; the Minkowski metric, $\eta_{\mu \nu}$, is then a good approximation to $g_{\mu \nu}$ in this neighborhood. However, as one deviates from this world line 
corrections to $\eta_{\mu \nu}$ appear, and since a specific coordinate system has been chosen, they appear as powers of the Riemann tensor (or its contractions), and its derivatives (see [22] and [23]). This means that the larger the curvature, the smaller the neighborhood about the world line where $\eta_{\mu \nu}$ is a good approximation of the metric. Consequently, the weak equivalence principle holds up to terms first order in the curvature. As the additional terms in Eq. (7) are first order in $R$ as well, our extension of the GEOM satisfies the weak equivalence principle to the same order of approximation as the GEOM does.

The strong equivalence principle Because we only change the geodesic Lagrangian, all nongravitational forces in our theory will have the same form as their special relativistic counterparts. Moreover, the extended GEOM reduces to the GEOM in the $R \rightarrow 0$ limit.

\subsection{Connections with other theories}

As unusual as the extended GEOM, Eq. (7), may appear to be, there are connections between this extension and other theories.

\subsubsection{The class of scalar field theories in curved spacetimes}

The Klein-Gordon equation corresponding to the extended GEOM is

$$
\nabla^{2} \phi+\frac{m^{2} c^{2}}{\hbar^{2}}\left[1+\mathfrak{D}\left(\frac{c^{2} R}{\Lambda_{\mathrm{DE}} G}\right)\right] \phi=0
$$

This is the equation of motion for a scalar field $\phi$ that is non-minimally and nonlinearly coupled to $R$. Scalar field theories of this class have been studied before, the most notable of which is

$$
\nabla^{2} \phi_{R}+\left(\frac{m^{2} c^{2}}{\hbar^{2}}+\xi R\right) \phi_{R}=0 .
$$

When $\xi=1 / 6$, the scalar field will be conformally invariant even though $m \neq 0$ [24].

There are important differences between these theories and the theory we are considering here, however. Scalar field theories of the form Eq. (14) were proposed at the quantum level and $\hbar$ appears explicitly; we are focused on the classical, $\hbar=0$, level. Note also that the scale of the coupling in Eq. (13) is set by $\ell_{\mathrm{DE}}$. This is a macroscopic in length scale, and if we expand $R$ about $4 \Lambda_{\mathrm{DE}} G / c^{2}$, Eq. (13) reduces to Eq. (14) with a

$$
\xi=\mathfrak{D}^{\prime}(4) \frac{m^{2} c^{2} \ell_{\mathrm{DE}}^{2}}{\hbar^{2}}
$$

which has a magnitude $\sim 10^{75}$ —indicative of an inherently classical theory-if $\phi$ has the mass of a proton. This value for $\xi$ is 75 orders of magnitude larger than the values 
of $\xi$ usually considered. It also signifies that a perturbative solution of Eq. (13) would be of limited use at best, and the non-linearity of the coupling must be explicitly taken into account.

\subsubsection{The $f(R)$ theory}

Proposals for modifying the Hilbert action by considering functions, $f(R)$, of the Ricci scalar have been made before (see [25] and [26] for reviews). These theories were first introduced to explain cosmic acceleration without the need for Dark Energy using a $1 / R$ action [27,28], and further extensions of this model have been made $[29,30]$ since then. They are now being studied in their own right, and various functional forms for $f(R)$ are being considered. Indeed, connection to Modified Newtonian Dynamics has been made for logarithmic $f(R)$ terms [31,32], while with other choices of $f(R)$ connection with quintessence has been made [33-38] as well. Importantly, issues with the introduction of a "fifth force", and compatibility with terrestrial experiments have begun to be addressed through the Chameleon Effect (see [39-42] and an overview in [33]), which is used to hide the effects of field with a small mass that would otherwise be seen.

It is also important to note that while $f(R)$ theories change the action for gravity, in our approach we do not; we still take the action for gravity to be the Hilbert action with the addition of a cosmological constant. We instead change the response of matter to gravity by extending the equations of motion for test particles, and thus change the energy-momentum tensor for matter.

\section{The energy-momentum tensor}

Beginning with [6], there have been a number of attempts to show that the GEOM are a necessary consequence of the Einstein's field equations, Eq. (1). Modern attempts at demonstrating such a linkage $[7,8]$ focus on the energy-momentum tensor, and consider the motion of a test particle moving in a region of spacetime where $T_{\mu \nu}=0$ outside of a "worldtube" that surround the test particle; inside this worldtube, Einstein tensor $G_{\mu \nu} \neq 0$. In fact, this tensor must satisfy the strong energy condition $G_{\mu \nu} t^{\mu} t^{\prime \nu} \leq 0$ (for our signature for the metric) there, where $t^{\mu}$ and $t^{\prime \nu}$ are two arbitrary, time-like vectors. As shown in [8], the test particle then necessarily moves along a geodesic. While this proof do not explicitly include the cosmological constant term, replacing $\tilde{G}_{\mu \nu}=G_{\mu \nu}+\Lambda_{\mathrm{DE}} g_{\mu \nu}$ does not materially change the nature of the proof given in [8]; since $\Lambda_{\mathrm{DE}}>0, \tilde{G} \mu \nu$ satisfies the strong energy condition as long as $G_{\mu \nu}$ does.

If $T_{\mu \nu}=0$ then $\mathfrak{R}\left[4+8 \pi T / \Lambda_{\mathrm{DE}}\right]=\mathfrak{R}[4]$, and is a constant. We then see explicitly from Eq. (7) that the extended GEOM reduces to the GEOM. As the $T_{\mu \nu}=0$ case is precisely the situation covered by [8], the extended GEOM does not violate these theorems. Indeed, in the following we will explicitly construct the energy-momentum tensor for dust within the extended GEOM framework.

Consider a collection of massive particles that can be treated as an inviscid fluid with density $\rho$, pressure $p$, and fluid velocity, $v^{\mu}(x)$. We consider the spacetime to be 
spatially symmetric, so that the most general form that the energy-momentum tensor for this fluid is the usual

$$
T_{\mu v}=\rho v_{\mu} v_{v}-\left(g_{\mu \nu}-\frac{v_{\mu} v_{v}}{c^{2}}\right) p .
$$

We emphasize that this form for $T_{\mu \nu}$ depends only on the spatial isotropy of the fluid, and thus holds for both the GEOM and the extended GEOM.

Following [22], energy and momentum conservation, $\nabla^{v} T_{\mu \nu}=0$, requires that

$$
0=v_{\nu} \nabla^{v}\left(\rho+p / c^{2}\right) v_{\mu}+\left(\rho+p / c^{2}\right) \nabla_{\nu} v^{\nu} v_{\mu}+\left(\rho+p / c^{2}\right) v^{\nu} \nabla_{\nu} v_{\mu}-\nabla_{\mu} p
$$

Since $v^{2}=$ constant even within the extended GEOM formulation, projecting the above along $v_{\mu}$ gives once again the first law of thermodynamics

$$
d\left(V \rho c^{2}\right)=-p d V
$$

where $V$ is the volume of the fluid. This analysis holds for both the GEOM and the extended GEOM, and thus the first law of thermodynamics holds for both equations of motion. The standard analysis of the evolution of the universe under the extended GEOM therefore follows much in the same way as before.

Next, projecting Eq. (17) along the subspace perpendicular to $v_{\mu}$ gives the relativistic version of Euler's equation

$$
0=\left(\rho+\frac{p}{c^{2}}\right) v^{\nu} \nabla_{\nu} v_{\mu}-\left(g_{\mu \nu}-\frac{v_{\mu} v_{\nu}}{c^{2}}\right) \nabla^{\nu} p
$$

Once again, Eq. (19) holds for both sets of equations of motion.

Consider now the simplest case when the constituent test particles in the fluid do not interact with one another except under gravity. This corresponds to the case of "dust". If test particles in this dust follow the GEOM, the solution to Eq. (19) gives the usual $T_{\mu \nu}^{\mathrm{GEOM}-\mathrm{Dust}}=\rho v_{\mu} v_{\nu}$ with $p \equiv 0$. If, on the other hand, the test particles follow the extended GEOM, the situation changes. Using Eq. (7), Eq. (19) becomes

$$
0=\left(g_{\mu \nu}-\frac{v_{\mu} v_{v}}{c^{2}}\right)\left\{\left(\rho c^{2}+p\right) \nabla^{v} \log \Re-\nabla^{v} p\right\}
$$

so that

$$
\left(\rho c^{2}+p\right) \nabla_{\mu} \mathfrak{R}-\mathfrak{R} \nabla_{\mu} p=\xi_{\mathrm{DE}} \Lambda_{\mathrm{DE}} c v_{\mu},
$$

where $\xi_{\mathrm{DE}}$ is a constant. By contracting the above with $v_{\mu}$, it is straightforward to see that if $\xi_{\mathrm{DE}} \neq 0, p$ will increase linearly with the proper time. This would be unphysical, and we conclude that $\xi_{\mathrm{DE}}$ must be zero.

Taking the pressure as a function of only the density, Eq. (21) reduces to a nonlinear, first order, ordinary differential equation. We will not solve this equation in general. Instead, we look at the nonrelativistic limit where $\rho c^{2} \gg 3 p$. Then $T \approx \rho c^{2}$, and 
$\Re$ can be approximated as a function of $\rho$ only. Equation (21) can then be solved implicitly to give

$$
p(\rho)=-\rho c^{2}+c^{2} \mathfrak{R}\left[4+8 \pi \rho / \Lambda_{\mathrm{DE}}\right] \int_{0}^{\rho} \frac{d s}{\Re\left[4+8 \pi s / \Lambda_{\mathrm{DE}}\right]} .
$$

Given the density, the pressure is then determined once a form for $\mathfrak{R}$ is known. The energy-momentum tensor for dust, $T^{\text {Ext-Dust }}$, under the extended GEOM can then be constructed using $\rho$, and the resultant $p$ from Eq. (22). Note also from Eq. (22) that $p \approx 4 \pi \mathfrak{R}^{\prime}[4] \rho^{2} c^{2} / \mathfrak{R}[4] \Lambda_{\mathrm{DE}}$ when $\rho \rightarrow 0$, while $p \approx-\Lambda_{\mathrm{DE}} c^{2}(1-1 / \mathfrak{R}[4])$ when $\rho \gg \Lambda_{\mathrm{DE}} / 2 \pi$. Thus, $T_{\mu \nu}^{\text {Ext-dust }} \approx \rho v_{\mu} v_{\nu}$, and the solution Eq. (22) is consistent with the approximation $T \approx \rho c^{2}$.

The physical reason for the presence of this pressure term in $T_{\mu \nu}^{\text {Ext-Dust }}$ can be seen from Eqs. (7) and (8). If the second equation is solved for the energy of a particle, it is straightforward to see that under the extended GEOM a collection of test particles behave as though they were in an external potential set by $\mathfrak{D}\left(c^{2} R / \Lambda_{\mathrm{DE}} G\right)$. As such, the particles no longer follow geodesics as they do in the GEOM even though they only interact with each other through gravity. The presence of the nonzero pressure term in $T_{\mu \nu}^{\text {Ext-Dust }}$ is a reflection of the presence of this potential.

\section{A form for $\mathfrak{D}(x)$ and experimental bounds on $\alpha_{\Lambda}$}

Our analysis up to now is valid for all $\mathfrak{D}(x)$. Requiring that our extension of the GEOM does not produce effects that would have already been observed in experiments will fix a specific form for $\mathfrak{D}(x)$.

Since our extension of the GEOM does not change the equations of motion for massless test particles, we expect Eq. (7) to reduce to the GEOM in the ultrarelativistic limit. It is thus only in the nonrelativistic limit where the effects of the deviations from the GEOM due to the additional terms in Eq. (7) can be seen. We therefore focus on the impact of the extension in the nonrelativistic, weak gravity limit, and begin by expressing Eq. (7) in these limits.

\subsection{Constructing $\mathfrak{D}(x)$}

We first perturb off the Minkowski metric $\eta_{\mu \nu}$ in the weak gravity limit by taking $g_{\mu \nu}=\eta_{\mu \nu}+h_{\mu \nu}$, where the only nonzero component of $h_{\mu \nu}$ is $h_{00}=2 \Phi / c^{2}$, and $\Phi$ is the gravitational potential. Equation (1) then gives

$$
\nabla^{2} \Phi+2 \frac{\Lambda_{\mathrm{DE}} G}{c^{2}} \Phi=4 \pi \rho G-\Lambda_{\mathrm{DE}} G
$$

in the presence of a cosmological constant. Next, the temporal coordinate, $x^{0}$, for the extended GEOM in this limit will, as usual, be approximated by $c t$ to lowest order 
in $|\mathbf{v}| / c$. The spatial coordinates, $\mathbf{x}$, on the other hand, reduce to

$$
\frac{d^{2} \mathbf{x}}{d t^{2}}=-\nabla \Phi-\left(\frac{4 \pi c^{2}}{\Lambda_{\mathrm{DE}}}\right)\left[\frac{\mathfrak{D}^{\prime}\left(4+8 \pi \rho / \Lambda_{\mathrm{DE}}\right)}{1+\mathfrak{D}\left(4+8 \pi \rho / \Lambda_{\mathrm{DE}}\right)}\right] \nabla \rho
$$

Here, we have assumed that the spacetime is spatially symmetric, and that the particle moves through an ambient, nonrelativistic fluid with density $\rho$.

For the additional terms in Eq. (24) from the extension not to contribute significantly to Newtonian gravity under current experimental conditions, $\mathfrak{D}^{\prime}\left(4+8 \pi \rho / \Lambda_{\mathrm{DE}}\right) \rightarrow 0$ when $\rho \gg \Lambda_{\mathrm{DE}} / 2 \pi$. We can now either choose $\mathfrak{D}^{\prime}(x)>0$ or $\mathfrak{D}^{\prime}(x)<0$, as $\mathfrak{D}^{\prime}(x) \rightarrow$ 0 from either above or below. This is an ambiguity in the construction of $\mathfrak{D}(x)$ that cannot be resolved with our current analysis; for now, we take $\mathfrak{D}^{\prime}(x)<0$. The simplest form for $\mathfrak{D}^{\prime}(x)$ with the correct asymptotic properties is

$$
\mathfrak{D}^{\prime}(x)=-\frac{\chi}{1+x^{1+\alpha_{\Lambda}}},
$$

where $\alpha_{\Lambda}$ is a power-law exponent that determines how fast $\mathfrak{D}^{\prime}(x) \rightarrow 0$ for $x \rightarrow \infty$, and $\chi$ is the normalization constant

$$
\frac{1}{\chi}=\int_{0}^{\infty} \frac{d s}{1+s^{1+\alpha_{\Lambda}}}
$$

To ensure nonzero effective masses, $\mathfrak{D}(x)$ must be positive, and we integrate Eq. (25) to get

$$
\mathfrak{D}(x)=\chi\left(\alpha_{\Lambda}\right) \int_{x}^{\infty} \frac{d s}{1+s^{1+\alpha_{\Lambda}}}
$$

with the condition that $\alpha_{\Lambda}>0$ to ensure that the the integral is defined. With this choice of integration constants, $\mathfrak{D}(0)=1$ and $\mathfrak{D}(x) \rightarrow 0$ as $x \rightarrow \infty$. While the precise form of $\mathfrak{D}(x)$ is calculable, we will not need it. Instead, because $8 \pi \rho / \Lambda_{\mathrm{DE}} \geq 0$,

$$
\mathfrak{D}\left(4+8 \pi \rho / \Lambda_{\mathrm{DE}}\right)=\chi \sum_{n=0}^{\infty} \frac{(-1)^{n}}{n\left(1+\alpha_{\Lambda}\right)+\alpha_{\Lambda}}\left(4+\frac{8 \pi \rho}{\Lambda_{\mathrm{DE}}}\right)^{-n\left(1+\alpha_{\Lambda}\right)-\alpha_{\Lambda}},
$$

while

$$
\frac{1}{\chi}=1+2 \sum_{n=0}^{\infty} \frac{(-1)^{n}}{\left[1+(n+1)\left(1+\alpha_{\Lambda}\right)\right]\left[n\left(1+\alpha_{\Lambda}\right)+\alpha_{\Lambda}\right]} .
$$

Notice that in the $\alpha_{\Lambda} \rightarrow \infty$ limit, $\mathfrak{D}(x) \rightarrow 0, \mathcal{L}_{\text {Ext }} \rightarrow \mathcal{L}_{0}$, and the GEOM is recovered. 
Bounds on $\alpha_{\Lambda}$ will be found below. For now, we note that for $\alpha_{\Lambda}>1, \chi \sim 1$ and $\mathfrak{D}\left(4+8 \pi \rho / \Lambda_{\mathrm{DE}}\right) \approx 0$. Thus,

$$
\frac{d^{2} \mathbf{x}}{d t^{2}} \approx-\nabla \Phi+\left(\frac{4 \pi c^{2} \chi}{\Lambda_{\mathrm{DE}}}\right)\left\{1+\left(4+\frac{8 \pi \rho}{\Lambda_{\mathrm{DE}}}\right)^{1+\alpha_{\Lambda}}\right\}^{-1} \nabla \rho
$$

\subsection{Constraints from terrestrial experiments}

We now consider the constraints placed on $\mathfrak{D}(x)$ due to terrestrial experiments.

From WMAP, $\Lambda_{\mathrm{DE}}=7.21_{-0.84}^{0.82} \times 10^{-30} \mathrm{~g} / \mathrm{cm}^{3}$, which for hydrogen atoms corresponds to a number density of $\sim 4$ atoms $/ \mathrm{m}^{3}$. It is clear that the density of both solids and liquids far exceed $\Lambda_{\mathrm{DE}}$, and in such media Eq. (30) reduces to what one expects for Newtonian gravity. Only very rare gases, in correspondingly hard vacuums, can have a density that is small enough for the additional terms in Eq. (24) to be relevant. The hardest vacuum currently attainable experimentally is $\sim 10^{-13}$ torr [43]. For a gas of $\mathrm{He}_{4}$ atoms at $4^{\circ} \mathrm{K}$, this corresponds to a density of $\rho_{\text {limit }} \approx 10^{-18} \mathrm{~g} / \mathrm{cm}^{3}$, which is 11 orders of magnitude smaller than $\Lambda_{\mathrm{DE}}$. Nevertheless, because the scale of the acceleration from the additional terms in Eq. (30)-which goes as $c^{2} / L$ for the relevant length scale $L$ in the experiment-is so large, effects at these densities will nevertheless be relevant. Indeed, we will use $\rho_{\text {limit }}$ to determine a lower bound to $\alpha_{\Lambda}$.

Consider a simple experiment that looks for signatures of the extension of the GEOM Eq. (30) by looking for anomalous accelerations (through pressure fluctuations) in a gas of $\mathrm{He}^{4}$ atoms at $4^{\circ} \mathrm{K}$ with a density $\rho_{\text {limit }}$. Inside this gas we consider a sound wave with amplitude $\epsilon \rho_{\text {limit }}$ propagating with a wavenumber $k$. Suppose that the smallest measurable acceleration for a test particle in this gas is $a_{\text {bound }}$. For the additional terms in Eq. (30) to be undetectable,

$$
a_{\text {bound }} \geq \frac{c^{2} \chi}{2}\left(\frac{\Lambda_{\mathrm{DE}}}{8 \pi \rho_{\text {limit }}}\right)^{\alpha_{\Lambda}} \epsilon k
$$

This gives a lower bound on $\alpha_{\Lambda}$ as

$$
\alpha_{\Lambda \text { bound }}=\frac{\log \left[2 a_{\text {bound }} / c^{2} \chi \epsilon k\right]}{\log \left[\Lambda_{\mathrm{DE}} / 8 \pi \rho_{\text {limit }}\right]} .
$$

For $\epsilon=0.1, k=1 \mathrm{~cm}^{-1}$, and $a_{\text {bound }}=1 \mathrm{~cm} / \mathrm{s}^{2}, \alpha_{\Lambda \text { bound }}$ ranges from 1.28 for $\Lambda_{\mathrm{DE}}=10^{-32} \mathrm{~g} / \mathrm{cm}^{3}$ to 1.58 for $\Lambda_{\mathrm{DE}}=10^{-29} \mathrm{~g} / \mathrm{cm}^{3}$. Notice that because $\alpha_{\Lambda \text { bound }}$ depends only logarithmically on $\epsilon, k$, and $a_{\text {bound }}$, the lower bound for $\alpha_{\Lambda}$ is relatively insensitive to the specific values taken for these parameters.

Adelberger has recently done state-of-the art, Eövtos-type experiments [44-46] to test the Newtonian, $1 / r^{2}$ law for gravity. While the pressures under which these experiments were performed were not explicitly stated, as far as we know these experiments were not done at pressures lower than $10^{-13}$ torr; we thus would not expect effects from extended GEOM to be apparent in these experiments either. 


\subsection{Constraints from solar system observations}

Most of the classical tests of general relativity are based on the motion of bodies in the solar system. We now consider the constraints placed on $\mathfrak{D}(x)$ due to these tests.

We begin by noting that in idealized situations such as the analysis of the advancement of perihelion of Mercury, the energy-momentum tensor is taken to be zero outside of a massive body such as the Sun; the terms on the right hand side of Eq. (7) will clearly not affect these analyses. While this argument would seem to hold for this and the other experimental tests of general relativity, it is much too simplistic. In practice, the $T_{\mu \nu}$ in each of these tests does not, in fact, vanish; a background density is always present. Except for experiments involving electromagnetic waves, what is instead needed is a comparison of the background density with $\Lambda_{\mathrm{DE}}$. It is only when this density is much greater than $\Lambda_{\mathrm{DE}} / 2 \pi$ that the additional terms in Eq. (7) may be negligible. We present this analysis below.

Of the classical, solar system tests of general relativity, only in observations or experiments involving motion of massive test particles can effects of the extension be seen. The extended GEOM does not affect the motion of electromagnetic waves, and such tests of general relativity as the gravitational redshift and the deflection of light by the Sun will not be affected by the extension. Considering first the advancement of the perihelion of Mercury, we note that the density of matter, $\rho_{\text {orb }}$, (due primarily to the solar wind) in the region of Mercury's orbit is $\sim 10^{-23} \mathrm{~g} / \mathrm{cm}^{3}$. This density is only five orders of magnitude smaller to $\rho_{\text {limit }}$. There is, then, a possibility that the extended GEOM could change the amount in which the perihelion of Mercury advances due to general relativistic effects.

To show that our extension nevertheless does not change the advance of perihelion, we follow extensively the derivation of the advancement of perihelion given in [22]. In particular, we use a Hamilton-Jacobi-based analysis to calculate the advancement and replace Eq. (8) by

$$
\begin{aligned}
m_{\mathrm{M}}^{2} c^{2}\left[1+\mathfrak{D}\left(4+\frac{8 \pi \rho_{\mathrm{orb}}}{\Lambda_{\mathrm{DE}}}\right)\right]= & {\left[1+2 \frac{G M_{\odot}}{c^{2} r}+2\left(\frac{G M_{\odot}}{c^{2} r}\right)^{2}\right]\left(\frac{\partial S_{\mathrm{M}}}{c \partial t}\right)^{2} } \\
& -\left[1-2 \frac{G M_{\odot}}{c^{2} r}\right]\left\{\left(\frac{\partial S_{\mathrm{M}}}{\partial r}\right)^{2}+\left(\frac{1}{r} \frac{\partial S_{\mathrm{M}}}{\partial \phi}\right)^{2}\right\}
\end{aligned}
$$

Here, $m_{\mathrm{M}}$ is the mass of Mercury, $M_{\odot}$ is the mass of the Sun, and $S_{\mathrm{M}}$ is the action for the motion of Mercury about the Sun. We have used the usual Schwarzchild solution for the region outside of the Sun, and kept terms up to second order in $G M / c^{2} r$. While in principal we would expect the extended GEOM to change the solution of Einstein's equations, we are working in both the weak gravity and nonrelativistic limits, where, as we have shown above, $T^{\text {Ext-Dust }} \approx \rho_{\text {orb }} c^{2}$; the Schwarzchild solution is unchanged in these limits. We also note that because $\rho_{\text {orb }} \sim 10^{-23} \mathrm{~g} / \mathrm{cm}^{3}$, the additional matter in Mercury's orbit will not affect the form of the Schwarzchild solution, and can be neglected when determining the metric outside of the Sun. 
We next look for solutions of the following form:

$$
S_{\mathrm{M}}=-m_{\mathrm{M}} c^{2} \tilde{E} t+m_{\mathrm{M}} \tilde{L} \varphi+m_{\mathrm{M}} c \mathfrak{S}(r)
$$

where $\tilde{E}$ is the energy per unit rest energy, $\tilde{L}$ the angular momentum per unit rest mass, $\varphi$ the azimuthal angle, and $r$ the radial position of Mercury measured from the Sun. Solution of Eq. (33) then gives

$$
\begin{aligned}
\mathfrak{S}= & \pm \int\left\{-\left(1+\mathfrak{D}-\tilde{E}^{2}\right)+2 \frac{G M}{c^{2} r}\left[1-2\left(1-\tilde{E}^{2}\right)\right]\right. \\
& \left.-\frac{\tilde{L}^{2}}{c^{2} r^{2}}+6\left(\frac{G M}{c^{2} r}\right)^{2}\right\}^{1 / 2} d r
\end{aligned}
$$

after keeping terms up to order $\left(G M_{\odot} / c^{2} r\right)^{2}$. In obtaining Eq. (35), we have used the fact that $1-\tilde{E}^{2} \sim G M_{\odot} / c^{2} a$, where $a$ is the semi-major axis of Mercury's orbit; observations set $a=57.91 \times 10^{6} \mathrm{~km}$ [47], giving $1-\tilde{E}^{2} \sim 10^{-8}$. We next used the relation from Eq. $(28), \mathfrak{D}\left[4+8 \pi \rho_{\text {orb }} / \Lambda_{\mathrm{DE}}\right] \approx\left(\chi / \alpha_{\Lambda}\right)\left(\Lambda_{\mathrm{DE}} / 8 \pi \rho_{\text {orb }}\right)^{\alpha_{\Lambda}}$. Thus, $\mathfrak{D} \sim 10^{-10}-10^{-12}$ for $\alpha_{\Lambda}=1.28-1.58$, so that $G M_{\odot} / c^{2} a<\mathfrak{D}<\left(G M_{\odot} / c^{2} a\right)^{2}$.

The shape of the orbit of Mercury is determined by minimizing the action, $\partial S / \partial \tilde{L}=$ 0, in Eq. (34). The resultant integral is straightforward to calculate, and we obtain

$$
\varphi=\left[1+3\left(\frac{G M_{\odot}}{c \tilde{L}}\right)^{2}\right] \cos ^{-1}\left[\frac{\left(1-e^{2}\right) a}{e r}-\frac{1}{e}\right]
$$

where analytically

$$
\begin{aligned}
a & =\frac{G M_{\odot}}{c^{2}} \frac{1-2\left(1-\tilde{E}^{2}\right)}{1+\mathfrak{D}-\tilde{E}^{2}} \\
1-e^{2} & =\left(\frac{c \tilde{L}}{G M_{\odot}}\right)^{2}\left(1+\mathfrak{D}-\tilde{E}^{2}\right)\left[1+4\left(1-\tilde{E}^{2}\right)-6\left(\frac{G M_{\odot}}{c \tilde{L}}\right)^{2}\right],
\end{aligned}
$$

and $e$ is the eccentricity of the orbit. The advancement in the perihelion of Mercury therefore still has the form

$$
\delta \varphi=\frac{6 \pi G M_{\odot}}{c^{2} a\left(1-e^{2}\right)} .
$$

Note, however, that the product $a\left(1-e^{2}\right)$ is independent of $\mathfrak{D}$. Thus, the amount that the perihelion of Mercury's orbit advances due to general relativistic effects is not changed by the extended GEOM. Physically, this is because the extended GEOM only modifies the rest mass in Eq. (8), and does not modify terms explicitly dependent on the velocity of Mercury. 
What the extended GEOM does affect is the analytical expressions for $e$ and $a$. However, in calculating the numerical value of $\delta \varphi$, both $e$ and $a$ are taken as measured quantities obtained from observations; they are not calculated from first principles. The fact that there is now a slight different relationship between $e$ and $a$, and the total energy, $m_{\mathrm{M}} c^{2} \tilde{E}$, and the angular momentum, $m_{\mathrm{M}} \tilde{L}$, of Mercury (by less than 0.01 $\%$ for $\alpha_{\Lambda}=1.58$ ) would require an independent method of determining $\tilde{E}$ and $\tilde{L}$ to check. Such an independent determination is not currently available.

There are three other potential solar system tests of general relativity that could, in principal, be used to constrain the extended GEOM. The first is looking at threebody effects in the lunar orbit due to the Sun, Earth and the Moon. It is questionable whether such effects can be measured [22], however, and they therefore cannot serve as a check on the extended GEOM. The second is frame dragging, where the precession of a spinning object is caused azimuthal changes in the local metric of spacetime by the rotating Earth; this effect is being measured by Gravity Probe B [48]. The third also measures frame dragging, but uses two satellites-LAGEOS and LAGEOS 2 -instead of a spinning body, to detect the Lense-Thirring effect; frame dragging was recently seen [49] in this experiment.

Frame dragging, whether it is by a spinning object or by two orbiting satellites, is inherently a velocity-dependent effect, however, that couples either to the spin of the object or to the orbital angular momentum of the satellites with the spacetime metric. Our extension of the GEOM changes the rest mass of the test particle, and in a spherically symmetric geometry in the nonrelativistic limit, the additional effects due to the extension is radial. We thus would not expect the effects from our extension can be seen either from the precession of a spin, or through the Lense-Thirring effect.

\section{Concluding remarks}

We have shown that because of the existence of a universal length scale, $\ell_{\mathrm{DE}}$, it is now possible to construct an extension of the GEOM. This extension preserves the equivalence principal, does not change the motion of massless test particles, and does not produce effects that would be detectable in either terrestrial experiments, or through observing the motion of bodies in the solar system. Our extension of the GEOM is thus a physically viable alternative to the GEOM.

The question remains as to whether these equations of motion have any physical relevance. In short, is anything gained by using this extension? Because $\ell_{\mathrm{DE}}=14010_{820}^{800}$ $\mathrm{Mpc}$, we would expect that any effects from the extended GEOM will become apparent at much longer length scales than those considered here. Indeed, given the size of $\ell_{\mathrm{DE}}$ the only reason why we would expect the extended GEOM to be relevant at all is because $\mathfrak{D}$ is a nonlinear function of the energy-momentum tensor of ambient matter. This question of relevance will be addressed in a future paper (A. D. Speliotopoulos, in preparation) where the extended GEOM is applied to the motion of stars in the rotation curves of galaxies, and to the density of matter at cosmological length scales. These are the scales at which we expect the effects from the extension to come into play, and where its relevance can be assessed.

Finally, as noted in [22], Eq. (19) can be solved in general to give an equation of motion for particles, $v^{v} \nabla_{\nu} v_{\mu}=\nabla_{\mu} p /\left(\rho+p / c^{2}\right)$, and we see that the presence of 
any pressure term in the energy-momentum tensor results in deviations from geodesic motion. Given that $\Lambda_{\mathrm{DE}}$ can also be used to construct a pressure, it is natural to ask whether the effects of the extended GEOM can be obtained through the introduction of an ad hoc pressure term in the energy-momentum tensor. Such an ad hoc term can only be introduced to the energy-momentum tensor for matter, however; for the reasons given in the introduction, the equations of motion for massless particles cannot be changed. In addition, because the behavior of any massive particle approaches that of a massless one in the ultrarelativistic limit, this ad hoc pressure term must be constructed so that irrespective of frame this term contributes a negligible amount to the energy of the particle in this limit. Moreover, even if such a construction can be accomplished at the ultrarelativistic limit, hurdles remain at the nonrelativistic limit. While it is possible to construct in the nonrelativistic limit an appropriate ad hoc pressure using $\Lambda_{\mathrm{DE}}$ and $\mathfrak{D}$, when the resultant equations of motion are applied to the same systems as the extended GEOM in (A. D. Speliotopoulos, in preparation), effects are predicted at the cosmological scale that either do not agree with experiment or are not physically reasonable. This occurs even though the pressure is chosen so that at galactic scales predicted effects will be in broad agreement with observations. For these reasons, it is doubtful that introducing ad hoc pressure term in place of the extended GEOM will be successful.

Acknowledgments The author would like to thank John Garrison for the numerous suggestions, comments, and the support he has given of his time while this research was being done. His efforts have helped guide it, and have elucidated many of the arguments given here. The author would also like to thank K.-W. Ng, H. T. Cho, and Clifford Richardson for their comments and criticisms while this research was done.

Open Access This article is distributed under the terms of the Creative Commons Attribution Noncommercial License which permits any noncommercial use, distribution, and reproduction in any medium, provided the original author(s) and source are credited.

\section{References}

1. Riess, A.G., Filippenko, A.V., Challis, P., Clocchiatti, A., Diercks, A., Garnavich, P.M., Gilliland, R.L., Hogan, C.J., Jha, S., Kirshner, R.P., Leibundgut, B., Phillips, M.M., Riess, D., Schmidt, B.P., Schommer, R.A., Smith, R.C., Spyromilio, J., Stubbs, C., Suntzeff, N.B., Tonry, J.: Astron. J. 116, 1009 (1998)

2. Perlmutter, S., Aldering, G., Goldhaber, G., Knop, R.A., Nugent, P., Castro, P.G., Deustua, S., Fabbro, S., Goobar, A., Groom, D.E., Hook, I.M., Kim, A.G., Kim, M.Y., Lee, J.C., Nunes, N.J., Pain, R., Pennypacker, C.R., Quimby, R., Lidman, C., Ellis, R., Irwin, M., McMahon, R.G., Ruiz-Lapuente, P., Walton, N., Schaefer, B., Boyle, B.J., Filippenko, A.V., Matheson, T., Fruchter, A.S., Panagia, N., Newberg, H.J.M., Couch, W.J.: Astrophys. J. 517, 565 (1999)

3. Bousso, R.: Gen. Relativ. Gravit. 40, 607 (2008)

4. Kapner, D.J., Cook, T.S., Adelberger, E.G., Gundlach, J.H., Heckel, B.R., Hoyle, C.D., Swanson, H.E.: Phys. Rev. Lett. 98, 021101 (2007)

5. Wald, R.: General Relativity, Chapter 3. The University of Chicago Press, Chicago (1984)

6. Einstein, A., Infield, L., Hoffmann, B.: Ann. Math. 39, 65 (1938)

7. Geroch, R., Jang, P.S.: J. Math. Phys. 16, 65 (1972)

8. Ehlers, J., Geroch, R.: gr-qc/0309074v1.

9. Milgrom, M.: Astrophys. J. 270, 365 (1983)

10. Milgrom, M.: Astrophys. J. 270, 371 (1983)

11. Milgrom, M.: Astrophys. J. 270, 384 (1983) 
12. Lämmerzahl, C., Preuss, O., Dittus, H.: gr-qc/0604052v1

13. Anderson, J.D., Laing, P.A., Lau, E.L., Liu, A.S., Nieto, M.M., Turyshev, S.G.: Phys. Rev. Lett. 81, 2858 (1998)

14. Anderson, J.D., Laing, P.A., Lau, E.L., Liu, A.S., Nieto, M.M., Turyshev, S.G.: Phys. Rev. D 65, 082004 (2002)

15. Anderson, J.D., Williams, J.G.: Class. Quantum Grav. 18, 2447 (2001)

16. Krasinsky, G.A., Brumberg, V.A.: Celest. Mech. Dyn. Astron. 90, 267 (2004)

17. Spergel, D.N., Bean, R., Doré, O., Nolta, M.R., Bennett, C.L., Dunkley, J., Hinshaw, G., Jarosik, N., Komatsu, E., Page, L., Peiris, H.V., Verde, L., Halpern, M., Hill, R.S., Kogut, A., Limon, M., Meyer, S.S., Odegard, N., Tucker, G.S., Weiland, J.L., Wollack, E., Wright, E.L.: Astrophys. J. Suppl. 170, 277 (2007)

18. Peebles, P.J.E., Ratra, B.: Astrophys. J. 325, L17 (1988)

19. Zlatev, I., Wang, L., Steinhardt, P.J.: Phys. Rev. Lett. 82, 896 (1999)

20. Armendariz-Picon, C., Mukhanov, V., Steinhardt, P.J.: Phys. Rev. Lett. 85, 4438 (2000)

21. Cahill, R.: arXiv:0709.2909 [physics.gen.ph]

22. Misner, C.W., Thorne, K.S., Wheeler, J.A.: Gravitation. W. H. Freeman and Company, San Francisco (1973)

23. Fermi E.: Atti. Accad. Naz. Lincei Rend. Cl. Sci. Fiz. Mat. Nat. Rend. 31, 21; 51 (1922)

24. Birrell, N.D., Davies, P.C.W.: Quantum Fields in Curved Space. Cambridge University Press, Cambridge (1982)

25. Navarro, I., van Acoleyen, K.: J. Cos. Astro. Phys. 3, 006 (2006)

26. Nojiri, S., Odintsov, S.D.: Int. J. Geom. Meth. Mod. Phys. 4, 115 (2007)

27. Capozziello, S., Troisi, A.: arXivLastro-ph/0303041

28. Carroll, S.M., Duvvuri, V., Trodden, M., Turner, M.S.: Phys. Rev. D 70, 043528 (2004)

29. Nojiri, S., Odintsov, S.D.: Phys. Rev. D 68, 123512 (2003)

30. Nojiri, S., Odintsov, S.D.: arXiv: 0707.1941 [hep-th]

31. Nojiri, S., Odintsov, S.D.: Gen. Relativ. Gravit. 36, 1765 (2004)

32. Navarro, I., van Acoleyen, K.: J. Cos. Astro. Phys. 9, 006 (2006)

33. Navarro, I., van Acoleyen, K.: J. Cos. Astro. Phys. 2, 022 (2007)

34. Whitt, B.: Phys. Lett. B 145, 176 (1984)

35. Barrow, J.D., Cotsakis, S.: Phys. Lett. B 214, 515 (1988)

36. Maeda, K.I.: Phys. Rev. D 39, 3159 (1989)

37. Wands, D.: Class. Quantum Grav. 11, 269 (1994)

38. Chiba, T.: Phys. Lett. B 575, 1 (2003)

39. Khoury, J., Weltman, A.: Phys. Rev. Lett. 93, 171104 (2004)

40. Khoury, J., Weltman, A.: Phys. Rev. D 69, 044026 (2004)

41. Brax, P., van de Bruck, C., Davis, A.C., Khoury, J., Weltman, A.: Phys. Rev. D 70, 123518 (2004)

42. Mota, D.F., Shaw, D.J.: Phys. Rev. Lett. 97, 151102 (2006)

43. Ishimura, H.: J. Vac. Sci. Technol. A 7, 3 (1978)

44. Hoyle, C.D., Schmidt, U., Heckel, B.R., Adelberger, E.G., Gundlach, J.H., Kapner, D.J., Swanson, H.E.: Phys. Rev. Lett. 86, 1418 (2001)

45. Adelberger, E.G., Heckel, B.R., Nelson, A.E.: Annu. Rev. Nucl. Part. Sci. 53, 77 (2003)

46. Hoyle, C.D., Kapner, D.J., Heckel, B.R., Adelberger, E.G., Gundlach, J.H., Schmidt, U., Swanson, H.E.: Phys. Rev. D 70, 042004 (2004)

47. http://nssdc.gsfc.nasa.gov/planetary/factsheet/mercuryfact.html

48. Fairbank, J.D., Dreaver, B.S. Jr., Everitt C.W.F., Michelson P.F. (eds.): In: Near Zero: New Frontiers of Physics. W. H. Freeman and Company, San Francisco (1988)

49. Ciufolini, I., Pavlis, E.C.: Nature 431, 958 (2004) 